\title{
Models of lightlike charges with nongeodesic worldlines
}

\author{
C. G. Böhmer* \\ Department of Mathematics, University College London, \\ Gower Street, London WCIE 6BT, United Kingdom \\ P. A. Hogan \\ School of Physics, University College Dublin, Belfield, Dublin 4, Ireland
}

(Received 13 June 2019; published 12 August 2019)

\begin{abstract}
Massless particles in general relativity move with the speed of light, and their trajectories in spacetime are described by null geodesics. This is independent of the electrical charge of the particle being considered; however, the charged lightlike case is less understood. Starting with the Maxwell field of a charged particle having a lightlike geodesic worldline in Minkowskian spacetime, we construct the Maxwell field of such a particle having a nongeodesic, lightlike worldline. The necessary geometry in the neighborhood of an arbitrary null worldline in Minkowskian spacetime is described and properties of the resulting electromagnetic field are discussed. The electromagnetic field obtained represents a lightlike analogue of the Liénard-Wiechert field, which generalizes the Coulomb field of a charge having a timelike geodesic worldline to the field of a charge having an accelerated worldline.
\end{abstract}

DOI: 10.1103/PhysRevD.100.044021

\section{INTRODUCTION}

It is an interesting and noteworthy fact that a charged particle traveling with the speed of light has yet to be observed in nature. There are no field theoretical considerations which would in principle contradict the existence of such a particle. Nevertheless, in exploring the limits of classical electrodynamics it is intriguing to seek models of such particles. This paper demonstrates explicitly that more than one model exists, and it will require further knowledge of the properties of such particles, if and when they are observed, to distinguish between them.

A particle with electrical charge $e$ having a timelike geodesic worldline in Minkowskian spacetime has a Maxwell field described by the Coulomb solution of the vacuum Maxwell field equations. If the worldline is not a geodesic, i.e. if the particle has nonvanishing 4-acceleration, then its Maxwell field is described by the Liénard-Wiechert solution of the vacuum Maxwell field equations. The Liénard-Wiechert electromagnetic field has the property that near the worldline of the charged particle it resembles the Coulomb field and far from the worldline it describes the electromagnetic radiation produced by the acceleration of the charge.

The Liénard-Wiechert 4-potential, when written in rectangular Cartesian coordinates and time, has the property of being proportional to the 4-velocity of the particle, modulo a

\footnotetext{
*c.boehmer@ucl.ac.uk

†peter.hogan@ucd.ie
}

gauge transformation. Many years ago Synge [1] looked for a Maxwell field of an accelerated lightlike charge by choosing a 4-potential proportional to the null tangent to the worldline of the charge. This resulted in an electromagnetic field which did not contain an analogue of the Coulomb part of the Liénard-Wiechert field but described electromagnetic radiation produced by the accelerated charge. If the charge has a null geodesic worldline, then the electromagnetic field vanishes. Charged gyratons which are models of massless charged particles with spin have been studied in [2]. This result suggests that it might also be possible to study charged lightlike particles using a mainly geometrical approach. This is the aim of the present paper. We seek to construct a model of an accelerated lightlike charge which incorporates an analogue of the Coulomb part of the Liénard-Wiechert field and an analogue of the radiation part of the Liénard-Wiechert field. Properties of hypothetical charged particles moving with the speed of light were already studied as early as the 1940s and were based on an entirely classical treatment; see in particular $[3,4]$.

We begin in Sec. II by describing the electromagnetic field of a charged particle having a null geodesic worldline. This is a lightlike analogue of the Coulomb field. The result is a spin-off from the Robinson-Trautman [5,6] solutions of the vacuum Einstein-Maxwell field equations. It exploits the idea of using null geodesics to set up a coordinate system which is ideally suited to the description of a lightlike particle. In Sec. III we develop the geometry associated with a nongeodesic, lightlike worldline in Minkowskian spacetime. A by-product of this study is to 
establish the existence of a parameter along the worldline which is unique up to a linear transformation and which specializes to an affine parameter if the worldline is a null geodesic. This is important because, in contradistinction to the timelike case, we do not have the arc length available to us as a parameter along the worldline in the lightlike case. The existence of such a parameter is one of the key ingredients of the final construction. Consequently, a model of the electromagnetic field of a lightlike charge, in the form of a solution of Maxwell's vacuum field equations on Minkowskian spacetime, is derived in Sec. IV, and some properties of the model are discussed in Sec. V. We conclude our work with discussions in the final section.

\section{LIGHTLIKE ANALOGUE OF THE COULOMB FIELD}

All topics under consideration in this paper are in the context of Minkowskian spacetime. The Minkowskian line element in rectangular Cartesian coordinates $X^{i}=$ $(T, X, Y, Z)$ reads

$d s^{2}=\eta_{i j} d X^{i} d X^{j}=(d T)^{2}-(d X)^{2}-(d Y)^{2}-(d Z)^{2}$.

We are working with signature $(+,-,-,-)$. The worldline in Minkowski space of a point charge giving rise to the Coulomb field is a timelike geodesic. For the lightlike analogue of the Coulomb field the worldline of the charge will be a null geodesic. We take this null geodesic to have parametric equations

$$
X^{i}(u)=u v^{i} \quad \text { with } \quad v^{i}=(1,0,0,1),
$$

where $v^{i}$ is a null vector because of $\eta_{i j} v^{i} v^{j}=v_{i} v^{i}=0$. The quantity $u$ is an affine parameter along the null geodesic with tangent $v^{i}$ and we can take $u \in \mathbb{R}$.

We will now introduce a new set of coordinates of the position 4-vector of a point in Minkowski space relative to this null geodesic as follows:

$$
X^{i}=u v^{i}+r k^{i}
$$

and we choose $k^{i}$ to satisfy

$$
k^{i} k_{i}=0 \quad \text { and } \quad k^{i} v_{i}=+1
$$

Hence, the worldline (2.2) corresponds to $r=0$, and we shall take $0 \leq r<+\infty$. This particular construction will prove very useful in the following as it is intimately tied to the geometry of a particle moving at the speed of light.

The vector $k^{i}$ is null and normalized relative to $v^{i}$ which means it can be parametrized by two real parameters $\xi \in \mathbb{R}$ and $\eta \in \mathbb{R}$. They determine the direction of $k^{i}$ in spacetime, and we can write this vector as

$$
k^{i}=\left(\frac{1}{2}\left(\xi^{2}+\eta^{2}+1\right), \xi, \eta, \frac{1}{2}\left(\xi^{2}+\eta^{2}-1\right)\right) .
$$

For sufficiently large values of $\xi$ and $\eta$ we write $\zeta=$ $\sqrt{\xi^{2}+\eta^{2}}$ and only keep terms in the highest power in $\zeta$. This gives

$$
k^{i} \rightarrow \frac{\zeta^{2}}{2}(1,0,0,1)=\frac{\zeta^{2}}{2} v^{i},
$$

for large $\zeta$. This means that $k^{i}$ points in the direction of $v^{i}$ in (2.2) for large $\xi$ and $\eta$.

Let us now consider (2.3) as a coordinate transformation between the original Cartesian coordinates and the new coordinates $x^{i}=(u, \xi, \eta, r)$. Writing this transformation out explicitly gives

$$
\begin{aligned}
& T=u+\frac{r}{2}\left(\xi^{2}+\eta^{2}+1\right), \\
& Z=u+\frac{r}{2}\left(\xi^{2}+\eta^{2}-1\right), \\
& X=r \xi, \quad Y=r \eta .
\end{aligned}
$$

Substituting (2.7)-(2.9) into the Minkowski line element (2.1) results in

$$
\begin{aligned}
d s^{2} & =2 d u d r-r^{2}\left(d \xi^{2}+d \eta^{2}\right) \\
& =2 \vartheta^{0} \vartheta^{3}-\left(\vartheta^{1}\right)^{2}-\left(\vartheta^{2}\right)^{2},
\end{aligned}
$$

with the basis 1 -forms $\vartheta^{0}, \vartheta^{1}, \vartheta^{2}, \vartheta^{3}$ given by

$\vartheta^{0}=d u, \quad \vartheta^{1}=r d \xi, \quad \vartheta^{2}=r d \eta, \quad \vartheta^{3}=d r$.

As the potential 1-form due to a particle of charge $e$ (which we assume to be constant) with worldline $r=0$, the lightlike analogue of the Coulomb potential, we take

$$
A=\frac{e}{r} \vartheta^{0}=\frac{e}{r} d u,
$$

where we emphasize that $u$ was the affine parameter along the null geodesic. The corresponding candidate for the Maxwell field due to this charged particle is the exterior derivative of $A$ resulting in the 2 -form

$$
F=d A=\frac{e}{r^{2}} d u \wedge d r=\frac{e}{r^{2}} \vartheta^{0} \wedge \vartheta^{3}
$$

The Hodge dual of $F$ is the 2 -form $* F$ given by

$$
* F=\frac{e}{r^{2}} \vartheta^{1} \wedge \vartheta^{2}=e d \xi \wedge d \eta,
$$

from which it immediately follows that Maxwell's vacuum field equations, 


$$
d * F=0
$$

are satisfied. Therefore, the potential 1-form (2.12) describes the Maxwell field of a lightlike particle and can be seen as the analogue of the Coulomb field of a timelike particle. The Maxwell field of such a lightlike particle is a spin-off of the charged Robinson-Trautman fields $[5,6]$ which are solutions of the vacuum EinsteinMaxwell field equations. It is worth pointing out that the entire construction of this solution was based on exploiting the inherent geometry of Minkowski space in the presence of particles moving at the speed of light.

\section{GEOMETRY ASSOCIATED WITH AN ACCELERATED LIGHTLIKE WORLDLINE}

We generalize the choice of coordinates (2.3) to a position 4-vector in Minkowskian spacetime relative to an arbitrary lightlike worldline with parametrization $X^{i}=w^{i}(u)$. The functions $w^{i}(u)$ were introduced to make the notation clearer and to distinguish from the previous case. The tangent vector to this curve is given by $v^{i}(u)=$ $d w^{i} / d u$ and satisfies $v_{i} v^{i}=0$, as before. Moreover, we introduce the acceleration $a^{i}(u)=d v^{i} / d u$ which satisfies $a_{i} v^{i}=0$. This follows from differentiating $v_{i} v^{i}=0$ with respect to the parameter $u$. In general $a^{i} \neq 0$ and so the lightlike worldline is not necessarily a geodesic and the particle having this worldline as its history will be said to be accelerated. The parameter $u$ along the worldline, for which $u \in \mathbb{R}$, is unspecified, and we will exploit this fact later. We replace (2.3) by the more general

$$
X^{i}=w^{i}(u)+r k^{i},
$$

with $k_{i} k^{i}=0$ and $k_{i} v^{i}=+1$. Hence the lightlike worldline corresponds to $r=0$. This setup is visualized in Fig. 1 .

As in the previous discussion, we parametrize the direction of $k^{i}$ with the two real parameters $x \in \mathbb{R}$ and $y \in \mathbb{R}$ and write

$-P_{0} k^{i}=\left(-1-\frac{1}{4}\left(x^{2}+y^{2}\right), x, y, 1-\frac{1}{4}\left(x^{2}+y^{2}\right)\right)$,

for some function $P_{0}(x, y, u)$. This function $P_{0}$ is determined by the normalization of the vector $k^{i}$ to be $v_{i} k^{i}=+1$ which means we have

$$
\begin{aligned}
P_{0}= & \left\{1+\frac{1}{4}\left(x^{2}+y^{2}\right)\right\} v^{0}(u)+x v^{1}(u)+y v^{2}(u) \\
& +\left\{1-\frac{1}{4}\left(x^{2}+y^{2}\right)\right\} v^{3}(u) .
\end{aligned}
$$

One should note that the components of the velocity are functions of the parameter $u$ only, and the spatial

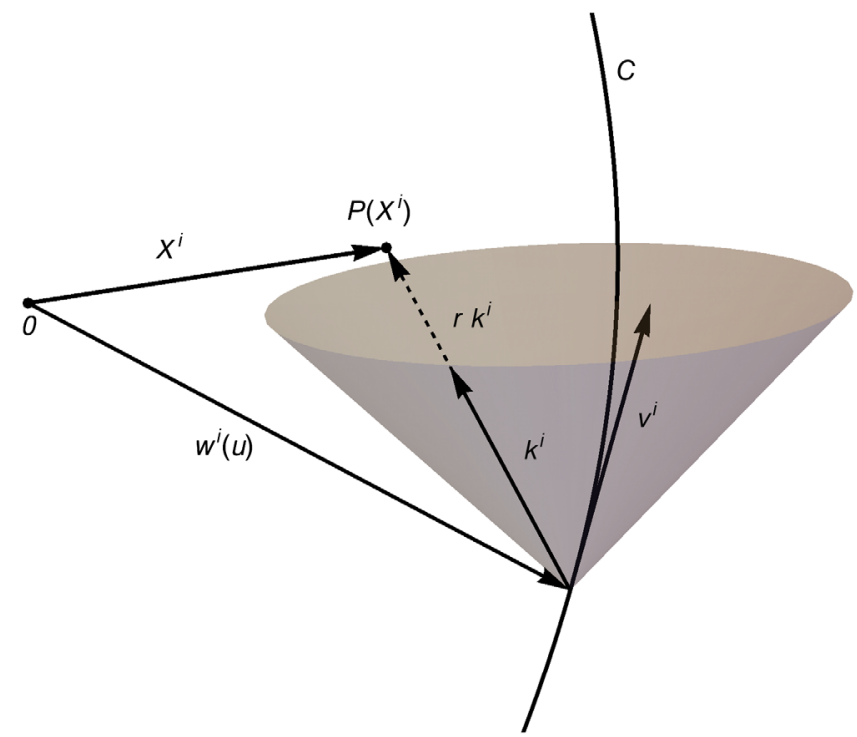

FIG. 1. The lightlike worldline (curve) is denoted by $C$, and its tangent vector is $v^{i} . k^{i}$ denotes the null vector and $r$ is the "distance" to the worldline. $X^{i}$ is the position vector of a point $P$ relative to the origin 0 .

dependence of $P_{0}$ enters entirely through the components of the vector $k^{i}$. A direct calculation shows that $P_{0}$ satisfies

$$
P_{0}^{2}\left(\frac{\partial^{2}}{\partial x^{2}}+\frac{\partial^{2}}{\partial y^{2}}\right) \log P_{0}=\Delta \log P_{0}=v^{i} v_{i}=0,
$$

where $\Delta$ stands for the covariant Laplacian on the 2-surface with line element $P_{0}^{-2}\left(d x^{2}+d y^{2}\right)$.

Contracting (3.2) with the acceleration vector yields the relation

$$
\begin{aligned}
P_{0} a_{i} k^{i}= & \left\{1+\frac{1}{4}\left(x^{2}+y^{2}\right)\right\} a^{0}(u)+x a^{1}(u)+y a^{2}(u) \\
& +\left\{1-\frac{1}{4}\left(x^{2}+y^{2}\right)\right\} a^{3}(u),
\end{aligned}
$$

and thus one can deduce that $P_{0}$ satisfies

$$
\frac{\partial}{\partial u} \log P_{0}=a_{i} k^{i}=: h_{0}
$$

which defines the new function $h_{0}$.

At this point we shall assume that $v^{0}-v^{3} \neq 0$. If $v^{0}=v^{3}$, then, since $v^{i}$ is a null vector, we have $v^{1}=0=v^{2}$ and also $a^{i}$ must be in the same direction [the $(T, Z)$-plane] as $v^{i}$; and so $r=0$ is a null geodesic, and we are led back to the situation discussed in Sec. II. So assuming from now on that $v^{0}-v^{3} \neq 0$, we can rewrite (3.3) in the useful form

$P_{0}=\frac{\left(v^{0}-v^{3}\right)}{4}\left\{\left(x+\frac{2 v^{1}}{v^{0}-v^{3}}\right)^{2}+\left(y+\frac{2 v^{2}}{v^{0}-v^{3}}\right)^{2}\right\}$. 
Note that this form of $P_{0}$ again shows directly that $\Delta \log P_{0}=0$. Following on from the previous construction, we introduce $(u, x, y, r)$ as coordinates, instead of $X^{i}$ which are related to the former by (3.1). The Minkowski line element (2.1) now takes the form

$$
d s^{2}=2 d u d r-2 h_{0} r d u^{2}-P_{0}^{-2}\left(d x^{2}+d y^{2}\right) .
$$

The form of $P_{0}$ given in (3.7) suggests a coordinate transformation from $x, y$ to $\xi, \eta$ given by

$$
\begin{gathered}
\xi=\frac{1}{P_{0}}\left(x+\frac{2 v^{1}}{v^{0}-v^{3}}\right), \\
\eta=\frac{1}{P_{0}}\left(y+\frac{2 v^{2}}{v^{0}-v^{3}}\right) .
\end{gathered}
$$

When these new coordinates are substituted into (3.8), the result is the line element

$$
\begin{aligned}
d s^{2}= & 2 d u d r-2 h_{0} r d u^{2} \\
& -r^{2}\left\{\left(d \xi+\frac{\partial q}{\partial \eta} d u\right)^{2}+\left(d \eta+\frac{\partial q}{\partial \xi} d u\right)^{2}\right\},
\end{aligned}
$$

with the function $q(\xi, \eta, u)$ given by

$$
\begin{aligned}
q(\xi, \eta, u)= & -\frac{1}{6}\left(\eta^{3} A^{1}+\xi^{3} A^{2}\right) \\
& +\xi \eta\left(\frac{1}{2}\left(\xi A^{1}+\eta A^{2}\right)+\frac{a^{0}-a^{3}}{v^{0}-v^{3}}\right)
\end{aligned}
$$

and

$A^{1}=a^{1}-\left(\frac{a^{0}-a^{3}}{v^{0}-v^{3}}\right) v^{1}, \quad A^{2}=a^{2}-\left(\frac{a^{0}-a^{3}}{v^{0}-v^{3}}\right) v^{2}$.

The null vector field $k^{i}$ in (3.2) written in terms of the parameters $\xi, \eta$ instead of $x, y$ can now be written in the form

$$
\begin{aligned}
k^{i} & =\zeta^{i}-\frac{1}{2}\left(\zeta_{k} \zeta^{k}\right) v^{i} \quad \text { with } \\
\zeta^{i} & =\left(\frac{1-\xi v^{1}-\eta v^{2}}{v^{0}-v^{3}},-\xi,-\eta, \frac{1-\xi v^{1}-\eta v^{2}}{v^{0}-v^{3}}\right) .
\end{aligned}
$$

Since $\zeta_{i} \zeta^{i}=-\left(\xi^{2}+\eta^{2}\right)=-\zeta^{2}$, we see that for large values of $\xi$ and $\eta$ the null vector $k^{i}$ points in the direction of the tangent $v^{i}$ to the worldline $r=0$, similar to the previous result (2.6). We also note that $q$ is a harmonic function and thus

$$
\Delta q=\frac{\partial^{2} q}{\partial \xi^{2}}+\frac{\partial^{2} q}{\partial \eta^{2}}=0
$$

and from (3.14) we have

$h_{0}=a_{i} k^{i}=\xi A^{1}+\eta A^{2}+\left(\frac{a^{0}-a^{3}}{v^{0}-v^{3}}\right)=\frac{\partial^{2} q}{\partial \xi \partial \eta}$.

At this point the parameter $u$ along the lightlike worldline $r=0$ is unspecified. It is useful to specify it up to a linear transformation as follows: Start with the coordinate transformation

$\bar{\xi}=\mu \xi, \quad \bar{\eta}=\mu \eta, \quad \bar{r}=\mu^{-1} r, \quad \bar{u}=\bar{u}(u)$,

with

$$
\mu^{-1} \frac{d \mu}{d u}=\frac{a^{0}-a^{3}}{v^{0}-v^{3}} \quad \text { and } \quad \frac{d \bar{u}}{d u}=\mu .
$$

We note from (3.18) that if we take

$$
\bar{u}=\int\left(v^{0}-v^{3}\right) d u,
$$

then $\bar{u}$ is unique up to a linear transformation $\bar{u} \rightarrow c_{1} \bar{u}+c_{2}$ where $c_{1}$ and $c_{2}$ are two real constants. If we let

$$
A^{i}=a^{i}-\left(\frac{a^{0}-a^{3}}{v^{0}-v^{3}}\right) v^{i},
$$

then the cases $i=1$ and $i=2$ are given in (3.13). If the worldline $r=0$ is a null geodesic, then, in general, $a^{i}=$ $\lambda(u) v^{i}$ for some function $\lambda(u)$ and $A^{i}=0$. The change of parameter $u$ along the worldline $r=0$ described by (3.18) results in

$$
v^{i}=\bar{v}^{i} \mu \quad \text { and } \quad a^{i}=\bar{v}^{i} \mu\left(\frac{a^{0}-a^{3}}{v^{0}-v^{3}}\right)+\bar{a}^{i} \mu^{2},
$$

where $\bar{v}^{i}=d w^{i} / d \bar{u}$ and $\bar{a}^{i}=d \bar{v}^{i} / d \bar{u}$. When this is substituted into (3.20) we find that

$$
A^{i}=\mu^{2} \bar{a}^{i}
$$

and so we have the result that

$$
a^{i}=\lambda(u) v^{i} \quad \Rightarrow \quad \bar{a}^{i}=0 .
$$

Hence we see an important property of the parameter $\bar{u}$, namely, if $r=0$ is a geodesic, then $\bar{u}$ is an affine parameter along it; see [7] for a similar discussion.

Now when the coordinate transformation (3.17) with (3.18) is applied to the line element (3.11) the result is

$$
\begin{aligned}
d s^{2}= & 2 d \bar{u} d \bar{r}-2 \bar{h}_{0} \bar{r} d \bar{u}^{2} \\
& -\bar{r}^{2}\left\{\left(d \bar{\xi}+\frac{\partial \bar{q}}{\partial \bar{\eta}} d \bar{u}\right)^{2}+\left(d \bar{\eta}+\frac{\partial \bar{q}}{\partial \bar{\xi}} d \bar{u}\right)^{2}\right\},
\end{aligned}
$$


with

$$
\bar{q}(\bar{\xi}, \bar{\eta}, \bar{u})=-\frac{1}{6}\left(\bar{\eta}^{3} \bar{a}^{1}+\bar{\xi}^{3} \bar{a}^{2}\right)+\frac{1}{2} \xi \eta\left(\bar{\xi} \bar{a}^{1}+\bar{\eta} \bar{a}^{2}\right)
$$

and

$$
\bar{h}_{0}=\frac{\partial^{2} \bar{q}}{\partial \bar{\xi} \partial \bar{\eta}} .
$$

Finally we note that since (3.20) implies that $A^{0}=A^{3}$, we have from (3.22) that $\bar{a}^{0}=\bar{a}^{3}$ because $\mu \neq 0$. These statements rely on our assumptions that $v^{0}-v^{3} \neq 0$. This, together with the orthogonality of $\bar{v}^{i}$ and $\bar{a}^{i}$, allows us to conclude that

$$
\bar{a}^{0}=\bar{a}^{3}=\frac{\bar{v}^{1}}{\bar{v}^{0}-\bar{v}^{3}} \bar{a}^{1}+\frac{\bar{v}^{2}}{\bar{v}^{0}-\bar{v}^{3}} \bar{a}^{2} .
$$

We are now able to apply these results to the study of an accelerated lightlike charge by following the exact same geometrical setup.

\section{ACCELERATED LIGHTLIKE CHARGE}

Guided by the analogue of the Coulomb solution of Maxwell's equations described in Sec. II, the work of Robinson and Trautman $[5,6]$ on solutions of the vacuum Einstein-Maxwell field equations, and requiring the solution of Maxwell's equations for the electromagnetic field of an accelerating lightlike charge to specialize to the case of an unaccelerated lightlike charge (2.12), we look for a potential 1-form to describe the Maxwell field of an accelerated lightlike charge of the form

$$
A=e\left(\frac{1}{\bar{r}}+G(\bar{\xi}, \bar{\eta}, \bar{u})\right) d \bar{u} .
$$

The aim is to specify the function $G(\bar{\xi}, \bar{\eta}, \bar{u})$ so that the Einstein-Maxwell equations are satisfied. Following on from the previously introduced basis 1-forms of the line element (3.24), we set

$$
\begin{array}{ccc}
\bar{\vartheta}^{0}=d \bar{u}, & \bar{\vartheta}^{3}=d \bar{r}-\bar{h}_{0} \bar{r} d \bar{u}, \\
\bar{\vartheta}^{1}=\bar{r}\left(d \bar{\xi}+\frac{\partial \bar{q}}{\partial \bar{\eta}} d \bar{u}\right), & \bar{\vartheta}^{2}=\bar{r}\left(d \bar{\eta}+\frac{\partial \bar{q}}{\partial \bar{\xi}} d \bar{u}\right) .
\end{array}
$$

The candidate for the Maxwell 2-form is the exterior derivative of (4.1) which reads

$$
\begin{aligned}
F & =d A=-\frac{e}{\bar{r}^{2}} d \bar{r} \wedge d \bar{u}+e \frac{\partial G}{\partial \bar{\xi}} d \bar{\xi} \wedge d \bar{u}+e \frac{\partial G}{\partial \bar{\eta}} d \bar{\eta} \wedge d \bar{u} \\
& =-\frac{e}{\bar{r}}\left(\frac{1}{\bar{r}} \bar{\vartheta}^{3}-\frac{\partial G}{\partial \bar{\xi}} \bar{\vartheta}^{1}-\frac{\partial G}{\partial \bar{\eta}} \bar{\vartheta}^{2}\right) \wedge \bar{\vartheta}^{0} .
\end{aligned}
$$

The Hodge dual of this 2-form becomes

$$
\begin{aligned}
* F= & \frac{e}{\bar{r}}\left(\frac{1}{\bar{r}} \bar{\vartheta}^{1} \wedge \bar{\vartheta}^{2}+\frac{\partial G}{\partial \bar{\xi}} \bar{\vartheta}^{2} \wedge \bar{\vartheta}^{0}-\frac{\partial G}{\partial \bar{\eta}} \bar{\vartheta}^{1} \wedge \bar{\vartheta}^{0}\right) \\
= & e d \bar{\xi} \wedge d \bar{\eta}+e\left(\frac{\partial \bar{q}}{\partial \bar{\xi}}-\frac{\partial G}{\partial \bar{\eta}}\right) d \bar{\xi} \wedge d \bar{u} \\
& -e\left(\frac{\partial \bar{q}}{\partial \bar{\eta}}-\frac{\partial G}{\partial \bar{\xi}}\right) d \bar{\eta} \wedge d \bar{u},
\end{aligned}
$$

from which one immediately arrives at

$d * F=e\left(-2 \frac{\partial^{2} \bar{q}}{\partial \bar{\xi} \partial \bar{\eta}}+\frac{\partial^{2} G}{\partial \bar{\xi}^{2}}+\frac{\partial^{2} G}{\partial \bar{\eta}^{2}}\right) d \bar{\xi} \wedge d \bar{\eta} \wedge d \bar{u}$.

Therefore Maxwell's vacuum field equations $d * F=0$ imply that $G$ must satisfy

$$
\Delta G=\frac{\partial^{2} G}{\partial \bar{\xi}^{2}}+\frac{\partial^{2} G}{\partial \bar{\eta}^{2}}=2 \frac{\partial^{2} \bar{q}}{\partial \bar{\xi} \partial \bar{\eta}}=2 \bar{h}_{0} .
$$

Since $\bar{q}$, given by (3.25), is also a harmonic function satisfying $\Delta \bar{h}_{0}=0$, it follows that $G$ satisfies the biharmonic equation

$$
\Delta \Delta G=0
$$

It is well known that the general solution of this equation is (a proof due to Schild is given in [8])

$G(\bar{\xi}, \bar{\eta}, \bar{u})=\operatorname{Re}\{f(\bar{\xi}+i \bar{\eta}, \bar{u})+(\bar{\xi}-i \bar{\eta}) F(\bar{\xi}+i \bar{\eta}, \bar{u})\}$,

where $f$ and $F$ are arbitrary analytic functions of $\bar{\xi}+i \bar{\eta}$. This means

$$
\begin{aligned}
f(\bar{\xi}+i \bar{\eta}, \bar{u}) & =U(\bar{\xi}, \bar{\eta}, \bar{u})+i V(\bar{\xi}, \bar{\eta}, \bar{u}) \quad \text { with } \\
\frac{\partial U}{\partial \bar{\xi}} & =\frac{\partial V}{\partial \bar{\eta}}, \quad \frac{\partial U}{\partial \bar{\eta}}=-\frac{\partial V}{\partial \bar{\xi}},
\end{aligned}
$$

and

$$
\begin{aligned}
F(\bar{\xi}+i \bar{\eta}, \bar{u}) & =W(\bar{\xi}, \bar{\eta}, \bar{u})+i S(\bar{\xi}, \bar{\eta}, \bar{u}) \quad \text { with } \\
\frac{\partial W}{\partial \bar{\xi}} & =\frac{\partial S}{\partial \bar{\eta}}, \quad \frac{\partial W}{\partial \bar{\eta}}=-\frac{\partial S}{\partial \bar{\xi}} .
\end{aligned}
$$

Hence (4.9) reads

$$
G(\bar{\xi}, \bar{\eta}, \bar{u})=U+\bar{\xi} W+\bar{\eta} S .
$$

We note in passing that we could equally well have used the imaginary part of $f+(\bar{\xi}+i \bar{\eta}) F$ for $G$ in (4.9). Clearly not all solutions of (4.8) are solutions of (4.7), and so substituting (4.12) into (4.7) yields 
$\Delta G=2\left(\frac{\partial W}{\partial \bar{\xi}}+\frac{\partial S}{\partial \bar{\eta}}\right)=4 \frac{\partial W}{\partial \bar{\xi}}=4 \frac{\partial S}{\partial \bar{\eta}}=2 \frac{\partial^{2} \bar{q}}{\partial \bar{\xi} \partial \bar{\eta}}$.

From this we have

$$
W=\frac{1}{2} \frac{\partial \bar{q}}{\partial \bar{\eta}}+\alpha(\bar{\eta}) \quad \text { and } \quad S=\frac{1}{2} \frac{\partial \bar{q}}{\partial \bar{\xi}}+\beta(\bar{\xi})
$$

where $\alpha$ and $\beta$ are functions of integration. But

$$
0=\frac{\partial W}{\partial \bar{\eta}}+\frac{\partial S}{\partial \bar{\xi}}=\frac{1}{2} \Delta \bar{q}+\frac{d \alpha}{d \bar{\eta}}+\frac{d \beta}{d \bar{\xi}}=\frac{d \alpha}{d \bar{\eta}}+\frac{d \beta}{d \bar{\xi}},
$$

since $\bar{q}$ is a harmonic function, and hence we must have

$$
\frac{d \alpha}{d \bar{\eta}}=C_{1}=-\frac{d \beta}{d \bar{\xi}} \Rightarrow \alpha(\bar{\eta})=C_{1} \bar{\eta}+C_{2}, \quad \beta(\bar{\xi})=-C_{1} \bar{\xi}+C_{3},
$$

where $C_{1}$ is a separation constant and $C_{2}$ and $C_{3}$ are constants of integration. Substituting (4.14) with (4.16) into (4.12) gives

$$
\begin{aligned}
G & =\underbrace{\frac{1}{2}\left(\bar{\xi} \frac{\partial \bar{q}}{\partial \bar{\eta}}+\bar{\eta} \frac{\partial \bar{q}}{\partial \bar{\xi}}\right)}_{\text {field of particle }}+\underbrace{U+C_{2} \bar{\xi}+C_{3} \bar{\eta}}_{\text {spherical EM waves }} \\
& =G_{\text {particle }}+G_{\text {spherical }} .
\end{aligned}
$$

The last three terms $G_{\text {spherical }}$ here constitute an arbitrary harmonic function, clearly $\Delta G_{\text {spherical }}=0$. When substituted into the Maxwell field (4.4) this harmonic function describes spherical electromagnetic waves which are independent of the accelerated lightlike particle and will therefore be excluded henceforth. The electromagnetic field of the lightlike particle is described simply by (4.4) with

$$
\begin{aligned}
G_{\text {particle }} & =\frac{1}{2}\left(\bar{\xi} \frac{\partial \bar{q}}{\partial \bar{\eta}}+\bar{\eta} \frac{\partial \bar{q}}{\partial \bar{\xi}}\right) \\
& =\frac{1}{4}\left(\bar{\xi}^{2}+\bar{\eta}^{2}\right)\left\{\bar{a}^{1}(u) \bar{\xi}+\bar{a}^{2}(u) \bar{\eta}\right\}
\end{aligned}
$$

If the worldline of the particle is a null geodesic, then $\bar{a}^{i}(\bar{u})=0$ and the Maxwell field of the accelerated lightlike particle specializes to the case described in Sec. II.

\section{PROPERTIES OF THE SOLUTION}

To obtain a useful comparison between the Maxwell field constructed from (4.4) and (4.18) and the Maxwell field of a point charge with a timelike worldline, we proceed as follows: Start by substituting the transformations (3.17) into the position vector (3.1) which becomes

$$
X^{i}=w^{i}(\bar{u})+\bar{r} \bar{k}^{i},
$$

where $\bar{k}^{i}$ is given by

$$
\begin{aligned}
& \bar{k}^{i}=\bar{q}^{i}-\frac{1}{2}\left(\bar{q}_{k} \bar{q}^{k}\right) \bar{v}^{i} \quad \text { with } \\
& \bar{q}^{i}=\left(\frac{1-\bar{\xi} \bar{v}^{1}-\bar{\eta} \bar{v}^{2}}{\bar{v}^{0}-\bar{v}^{3}},-\bar{\xi},-\bar{\eta}, \frac{1-\bar{\xi} \bar{v}^{1}-\bar{\eta} \bar{v}^{2}}{\bar{v}^{0}-\bar{v}^{3}}\right) .
\end{aligned}
$$

Geometrically, this is the same setup as shown in Fig. 1 with all quantities replaced by their barred counterparts. From this $\bar{k}^{i}$ we calculate the useful formulas

$$
\frac{\partial \bar{k}^{i}}{\partial \bar{\xi}} \frac{\partial \bar{k}_{i}}{\partial \bar{\xi}}=\frac{\partial \bar{k}^{i}}{\partial \bar{\eta}} \frac{\partial \bar{k}_{i}}{\partial \bar{\eta}}=-1, \quad \frac{\partial \bar{k}^{i}}{\partial \bar{\xi}} \frac{\partial \bar{k}_{i}}{\partial \bar{\eta}}=0,
$$

and

$$
\frac{\partial \bar{k}^{i}}{\partial \bar{\xi}} \frac{\partial \bar{k}_{i}}{\partial \bar{u}}=-\frac{\partial \bar{q}}{\partial \bar{\eta}}, \quad \frac{\partial \bar{k}^{i}}{\partial \bar{\eta}} \frac{\partial \bar{k}_{i}}{\partial \bar{u}}=-\frac{\partial \bar{q}}{\partial \bar{\xi}} .
$$

Consequently we can rearrange to find

$$
\frac{\partial \bar{k}^{i}}{\partial \bar{u}}=-\bar{h}_{0} \bar{k}^{i}+\frac{\partial \bar{q}}{\partial \bar{\eta}} \frac{\partial \bar{k}^{i}}{\partial \bar{\xi}}+\frac{\partial \bar{q}}{\partial \bar{\xi}} \frac{\partial \bar{k}^{i}}{\partial \bar{\eta}} .
$$

Equation (5.1) implicitly defines $\bar{\xi}, \bar{\eta}, \bar{r}, \bar{u}$ as functions of $X^{i}$. The gradients of these functions are obtained by first differentiating (5.1) with respect to $X^{j}$ to find that

$\delta_{j}^{i}=\left(\bar{v}^{i}+\bar{r} \frac{\partial \bar{k}^{i}}{\partial \bar{u}}\right) \bar{u}_{, j}+\bar{k}^{i} \bar{r}_{, j}+\bar{r} \frac{\partial \bar{k}^{i}}{\partial \bar{\xi}} \bar{\xi}_{, j}+\bar{r} \frac{\partial \bar{k}^{i}}{\partial \bar{\eta}} \bar{\eta}_{, j}$.

Multiplying this equation successively by $\bar{k}_{i}, \bar{v}_{i}, \partial \bar{k}_{i} / \partial \bar{\xi}$, $\partial \bar{k}_{i} / \partial \bar{\eta}$ and using (5.3) and (5.4) results in

$\bar{u}_{, j}=\bar{k}_{j}, \quad \bar{r}_{, j}=\bar{v}_{j}+\bar{r}_{h_{0}} \bar{k}_{j}$,

$\bar{\xi}_{, j}=-\frac{1}{\bar{r}} \frac{\partial \bar{k}_{j}}{\partial \bar{\xi}}-\frac{\partial \bar{q}}{\partial \bar{\eta}} \bar{k}_{j}, \quad \bar{\eta}_{, j}=-\frac{1}{\bar{r}} \frac{\partial \bar{k}_{j}}{\partial \bar{\eta}}-\frac{\partial \bar{q}}{\partial \bar{\xi}} \bar{k}_{j}$.

Now the potential 1-form (4.1) with (4.18) is equivalent to the 4-potential in coordinates $X^{i}$ :

$$
A_{i}=e\left(\frac{1}{\bar{r}}+G(\bar{\xi}, \bar{\eta}, \bar{u})\right) \bar{k}_{i} .
$$

The Maxwell field (4.4) has components $F_{i j}=-F_{j i}$ in coordinates $X^{i}$ given by 


$$
\begin{aligned}
F_{i j}= & A_{j, i}-A_{i, j} \\
= & \frac{e}{\bar{r}^{2}}\left(\bar{k}_{i} \bar{v}_{j}-\bar{k}_{j} \bar{v}_{i}\right) \\
& +\frac{e}{\bar{r}}\left\{\frac{\partial G}{\partial \bar{\xi}}\left(\bar{k}_{i} \frac{\partial \bar{k}_{j}}{\partial \bar{\xi}}-\bar{k}_{j} \frac{\partial \bar{k}_{i}}{\partial \bar{\xi}}\right)+\frac{\partial G}{\partial \bar{\eta}}\left(\bar{k}_{i} \frac{\partial \bar{k}_{j}}{\partial \bar{\eta}}-\bar{k}_{j} \frac{\partial \bar{k}_{i}}{\partial \bar{\eta}}\right)\right\} .
\end{aligned}
$$

The dual of this Maxwell field has components $* F_{i j}=\frac{1}{2} \epsilon_{i j k l} F^{k l}$, with $\epsilon_{i j k l}$ the Levi-Cività permutation symbol in four dimensions, and these components are given by

$$
\begin{aligned}
* F_{i j}= & \frac{e}{\bar{r}^{2}}\left(\frac{\partial \bar{k}_{i}}{\partial \bar{\xi}} \frac{\partial \bar{k}_{j}}{\partial \bar{\eta}}-\frac{\partial \bar{k}_{i}}{\partial \bar{\eta}} \frac{\partial \bar{k}_{j}}{\partial \bar{\xi}}\right) \\
& +\frac{e}{\bar{r}}\left\{\frac{\partial G}{\partial \bar{\xi}}\left(\bar{k}_{i} \frac{\partial \bar{k}_{j}}{\partial \bar{\eta}}-\bar{k}_{j} \frac{\partial \bar{k}_{i}}{\partial \bar{\eta}}\right)-\frac{\partial G}{\partial \bar{\eta}}\left(\bar{k}_{i} \frac{\partial \bar{k}_{j}}{\partial \bar{\xi}}-\bar{k}_{j} \frac{\partial \bar{k}_{i}}{\partial \bar{\xi}}\right)\right\}
\end{aligned}
$$

which is equivalent to (4.5). We note that

$$
F_{i j} \bar{k}^{j}=\frac{e}{\bar{r}^{2}} \bar{k}_{i} \quad \text { and } \quad * F_{i j} \bar{k}^{j}=0
$$

Hence the field (5.9) is qualitatively similar, from an algebraic point of view, to the electromagnetic field of an accelerated point charge traveling with less than the speed of light. The $\bar{r}^{-2}$-part of the field is algebraically general and corresponds to the Coulomb part of the field in the timelike case. The $\bar{r}^{-1}$-part of the field is algebraically special (purely radiative) with degenerate principal null direction $\bar{k}^{i}$. It describes the electromagnetic radiation produced by the accelerated source just as in the timelike case. Unlike the timelike case the field here is singular at $\bar{r}=0$ (on the null worldline of the source) and at $\bar{\xi}, \bar{\eta} \rightarrow \infty$, on account of (4.18), which by (5.2) corresponds to $\bar{k}^{i}$ pointing along the direction of the tangent $\bar{v}^{i}$ to the source worldline.

Finally it is interesting to compare the model of an accelerated lightlike charge described here with the model given by Synge [1]. In our formalism Synge's potential 1 -form is

$$
A=-e \bar{h}_{0} d \bar{u} \text { with } \bar{h}_{0}=\bar{a}_{i} \bar{k}^{i}=\bar{a}^{1}(\bar{u}) \bar{\xi}+\bar{a}^{2}(\bar{u}) \bar{\eta} .
$$

The corresponding Maxwell field and its dual are given by the 2-forms

$$
\begin{aligned}
F & =-\frac{e}{\bar{r}} \frac{\partial \bar{h}_{0}}{\partial \bar{\xi}} \bar{\vartheta}^{1} \wedge \bar{\vartheta}^{4}-\frac{e}{\bar{r}} \frac{\partial \bar{h}_{0}}{\partial \bar{\eta}} \bar{\vartheta}^{2} \wedge \bar{\vartheta}^{4} \\
& =-\frac{e}{\bar{r}}\left\{\bar{a}^{1} \bar{\vartheta}^{1} \wedge \bar{\vartheta}^{4}+\bar{a}^{2} \bar{\vartheta}^{2} \wedge \bar{\vartheta}^{4}\right\}
\end{aligned}
$$

and

$$
\begin{aligned}
* F & =\frac{e}{\bar{r}}\left\{-\bar{a}^{1} \bar{\vartheta}^{2} \wedge \bar{\vartheta}^{4}+\bar{a}^{2} \bar{\vartheta}^{1} \wedge \bar{\vartheta}^{4}\right\} \\
& =e\left\{-\bar{a}^{1} d \bar{\eta} \wedge d \bar{u}+\bar{a}^{2} d \bar{\xi} \wedge d \bar{u}\right\} .
\end{aligned}
$$

From the latter it is clear that Maxwell's vacuum field equations $d * F=0$ are satisfied. If the worldline $\bar{r}=0$ is a null geodesic, then Synge's Maxwell field vanishes and there is no lightlike analogue of the Coulomb field in his case. In terms of the coordinates $X^{i}$ the components $F_{i j}$ of the Maxwell field (5.13) and the components $* F_{i j}$ of its dual (5.14) read

$F_{i j}=\frac{e}{\bar{r}}\left\{\left(\frac{\partial \bar{h}_{0}}{\partial \bar{\xi}} \frac{\partial \bar{k}_{i}}{\partial \bar{\xi}}+\frac{\partial \bar{h}_{0}}{\partial \bar{\eta}} \frac{\partial \bar{k}_{i}}{\partial \bar{\eta}}\right) \bar{k}_{j}-\left(\frac{\partial \bar{h}_{0}}{\partial \bar{\xi}} \frac{\partial \bar{k}_{j}}{\partial \bar{\xi}}+\frac{\partial \bar{h}_{0}}{\partial \bar{\eta}} \frac{\partial \bar{k}_{j}}{\partial \bar{\eta}}\right) \bar{k}_{i}\right\}$

and

$* F_{i j}=\frac{e}{\bar{r}}\left\{\left(\frac{\partial \bar{h}_{0}}{\partial \bar{\xi}} \frac{\partial \bar{k}_{i}}{\partial \bar{\eta}}-\frac{\partial \bar{h}_{0}}{\partial \bar{\eta}} \frac{\partial \bar{k}_{i}}{\partial \bar{\xi}}\right) \bar{k}_{j}-\left(\frac{\partial \bar{h}_{0}}{\partial \bar{\xi}} \frac{\partial \bar{k}_{j}}{\partial \bar{\eta}}-\frac{\partial \bar{h}_{0}}{\partial \bar{\eta}} \frac{\partial \bar{k}_{j}}{\partial \bar{\xi}}\right) \bar{k}_{i}\right\}$,

respectively. From these we have

$$
F_{i j} \bar{k}^{j}=0=* F_{i j} \bar{k}^{j}
$$

indicating that the Maxwell field is pure electromagnetic radiation with propagation direction $\bar{k}^{i}$ in Minkowskian spacetime. When (5.7) are substituted into (5.6) using (5.5) and raising the covariant index, we have

$$
\eta^{i j}=-\frac{\partial \bar{k}^{i}}{\partial \bar{\xi}} \frac{\partial \bar{k}^{j}}{\partial \bar{\xi}}-\frac{\partial \bar{k}^{i}}{\partial \bar{\eta}} \frac{\partial \bar{k}^{j}}{\partial \bar{\eta}}+\bar{v}^{i} \bar{k}^{j}+\bar{v}^{j} \bar{k}^{i}
$$

Hence with $\bar{h}_{0}$ given by (5.12) we can write

$$
\begin{aligned}
\frac{\partial \bar{h}_{0}}{\partial \bar{\xi}} \frac{\partial \bar{k}_{i}}{\partial \bar{\xi}}+\frac{\partial \bar{h}_{0}}{\partial \bar{\eta}} \frac{\partial \bar{k}_{i}}{\partial \bar{\eta}} & =\bar{a}^{j}\left(\frac{\partial \bar{k}_{j}}{\partial \bar{\xi}} \frac{\partial \bar{k}_{i}}{\partial \bar{\xi}}+\frac{\partial \bar{k}_{j}}{\partial \bar{\eta}} \frac{\partial \bar{k}_{i}}{\partial \bar{\eta}}\right) \\
& =\bar{h}_{0} \bar{v}_{i}-\bar{a}_{i},
\end{aligned}
$$

and so we can simplify (5.15) to the form given originally by Synge:

$$
F_{i j}=\frac{e}{\bar{r}}\left\{\bar{k}_{i} \bar{a}_{j}-\bar{k}_{j} \bar{a}_{i}+\bar{h}_{0}\left(\bar{v}_{i} \bar{k}_{j}-\bar{v}_{j} \bar{k}_{i}\right)\right\} .
$$

From the first of (5.7) Synge's potential 1-form in coordinates $X^{i}$ reads

$$
A=-e \bar{h}_{0} \bar{k}_{i} d X^{i}=A_{i} d X^{i} .
$$

Using the second of (5.7) we can write the 4-potential here as 


$$
A_{i}=e \frac{\bar{v}_{i}}{\bar{r}}-e(\log \bar{r})_{, i},
$$

demonstrating that, modulo a gauge term, the 4-potential has the same algebraic form as the Liénard-Wiechert 4potential in the timelike case. In other words it is pointing in the direction of the tangent to the worldline of the source.

Let us have a closer look at the electric and magnetic fields encoded in the Faraday tensor (5.20). To do so we introduce the notation $\boldsymbol{a}=a_{\alpha}, \alpha=1,2,3$, for the purely spatial part of the 4-vector $a_{i}$. It will prove useful to introduce the rescaled vector $\boldsymbol{K}=\overline{\boldsymbol{k}} / \bar{k}_{0}$. One can read off the electric field directly as $E_{\alpha}=F_{0 \alpha}$ so that

$$
\begin{aligned}
\boldsymbol{E} & =\frac{e}{\bar{r}}\left\{\bar{k}_{0}\left(\overline{\boldsymbol{a}}-\bar{h}_{0} \overline{\boldsymbol{v}}\right)-\left(\bar{a}_{0}-\bar{h}_{0} \bar{v}_{0}\right) \overline{\boldsymbol{k}}\right\} \\
& =\frac{e}{\bar{r}}\left\{\left(\overline{\boldsymbol{a}}-\bar{h}_{0} \overline{\boldsymbol{v}}\right)-\left(\bar{a}_{0}-\bar{h}_{0} \bar{v}_{0}\right) \boldsymbol{K}\right\} \bar{k}_{0} .
\end{aligned}
$$

Likewise, we find for the magnetic field the expression

$$
\boldsymbol{B}=\frac{e}{\bar{r}}\left\{\left(\overline{\boldsymbol{a}}-\bar{h}_{0} \overline{\boldsymbol{v}}\right) \times \boldsymbol{k}\right\}=\frac{e}{\bar{r}}\left\{\left(\overline{\boldsymbol{a}}-\bar{h}_{0} \overline{\boldsymbol{v}}\right) \times \boldsymbol{K}\right\} \bar{k}_{0},
$$

which yields the somewhat expected relations

$$
\begin{array}{ll}
\boldsymbol{E} \cdot \boldsymbol{B}=0, & |\boldsymbol{E}|=|\boldsymbol{B}|=\frac{e}{\bar{r}} \sqrt{-\bar{a}_{i} \bar{a}^{i}} \bar{k}_{0}, \\
\boldsymbol{E} \cdot \boldsymbol{K}=0, & \boldsymbol{E} \times \boldsymbol{K}=\boldsymbol{B} \quad(\Rightarrow \boldsymbol{B} \cdot \boldsymbol{K}=0) .
\end{array}
$$

This means we are dealing with an electromagnetic wave propagating along the direction defined by the spatial vector $\boldsymbol{K}$. Note that this vector is normalized to unity which follows from the fact that the original 4-vector $k_{i}$ was null. Hence these waves are traveling at the speed of light. As mentioned above, the electromagnetic field vanishes when the acceleration vanishes which corresponds to $\bar{r}=0$ being a geodesic worldline. In [7] a similar observation was made where $\bar{a}^{i}=d^{2} w^{i} / d u^{2}$ was interpreted as the curvature of the worldline $w^{i}(u)$.

\section{CONCLUSIONS OR DISCUSSIONS}

The model of a lightlike charge with a nongeodesic worldline given here places a great emphasis on geometry. The geometry involved utilizes a construction in the vicinity of an arbitrary lightlike worldline in Minkowskian spacetime. This geometrical approach allows us to study charged particles moving with the speed of light directly. A key ingredient is the choice of parameter along such a worldline since the natural parameter of arc length in the timelike case is not available in the lightlike case. We have demonstrated in Sec. III the existence of a special parameter $\bar{u}$ along the lightlike worldline, unique up to a linear transformation, having the useful property in the current context that it becomes an affine parameter when the worldline is a null geodesic. This appears to be a natural choice of parameter for the construction given in this paper which then allows us to construct the electric and magnetic fields of such a hypothetical particle. Our approach allows this construction without the need to introduce sources.

Interestingly Synge [9] has pointed out that "not quite obviously there exists on the curve a canonical parameter $u$ such that $a^{i} a_{i}=-1$ with $u$ defined to within an additive constant." Synge's choice of parameter is very interesting and may be the natural choice for scenarios other than that considered here.
[1] J. L. Synge, Tensor 24, 69 (1972).

[2] V. P. Frolov and A. Zelnikov, Classical Quantum Gravity 23, 2119 (2006).

[3] J. Weyssenhoff and A. Raabe, Acta Phys. Pol. B IX, 7 (1947).

[4] J. Weyssenhoff and A. Raabe, Acta Phys. Pol. B IX, 19 (1947).

[5] I. Robinson and A. Trautman, Phys. Rev. Lett. 4, 431 (1960).
[6] I. Robinson and A. Trautman, Proc. R. Soc. A 265, 463 (1962).

[7] P. O. Kazinski and A. A. Sharapov, Classical Quantum Gravity 20, 2715 (2003).

[8] J. L. Synge, The Hypercircle in Mathematical Physics (Cambridge University Press, Cambridge, England, 1957), p. 355.

[9] J. L. Synge (private communication). 